WIS $-97 / 7 /$ Feb $-\mathrm{PH}$

\title{
Rate equations for quantum transport in multi-dot systems
}

\author{
S.A. Gurvitz \\ Department of Particle Physics, Weizmann Institute of Science, Rehovot 76100, Israel
}

(October 10, 2018)

\begin{abstract}
Starting with the many-body Schrödinger equation we derive new rate equations for resonant transport in quantum dots linked by ballistic channels with high density of states. The charging and the Pauli exclusion principle effects were taken into account. It is shown that the current in such a system displays quantum coherence effects, even if the dots are away one from another. A comparative analysis of quantum coherence effects in coupled and separated dots is presented. The rate equations are extended for description of coherent and incoherent transport in arbitrary multi-dot systems. It is demonstrated that new rate equations constitute a generalization of the well-known optical Bloch equations.
\end{abstract}

\section{INTRODUCTION}

Quantum transport in small tunneling structures (quantum dots) have attracted great attention due to the possibility of investigating single-electron effects in the electric current [1]. Until now research has been mostly concentrated on single dots, but the rapid progress in microfabrication technology has made it possible the extension to coupled dot systems with aligned levels [2 [1. An example of such a system is shown schematically in Fig. 1, where the coupled quantum dot (coupled wells) are connected with two separate reservoirs (the leads). 
In contrast with a single dot, the electron wave function inside a coupled dot structure is a superposition of electron states localized in each of the dots. As a result, the effects of quantum coherence would appear in electron current flowing through such a system. Usually these effects are treated in the framework of single electron approach. Although this approach is an appropriate one for coupled-well semiconductor heterostructures [5 7], it cannot be applied for coupled dots. The reason is the dominant role of the Coulomb interaction that leads to the Hubbard-type Hamiltonian for a description of these systems 8.

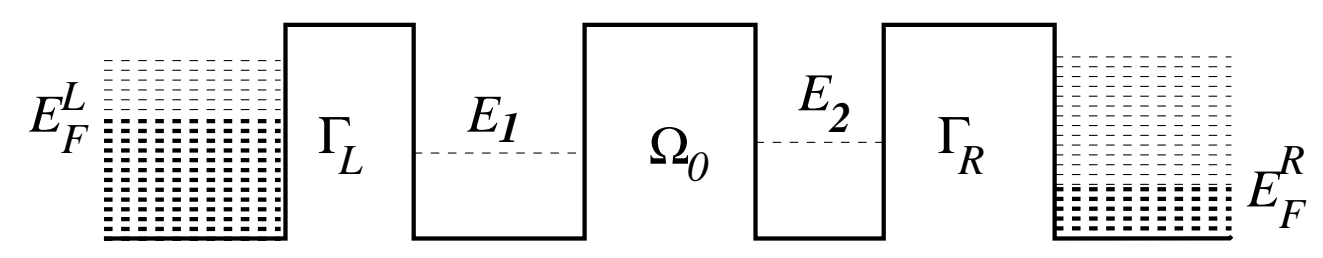

Fig. 1. Resonant transport through a coupled-dot structure. $\Gamma_{L, R}$ denote the corresponding tunneling rate from (to) the left (right) reservoirs, and $\Omega_{0}$ is the interdot hopping amplitude. $E_{F}^{L, R}$ denote the Fermi level in the left (right) reservoirs. Only those energy levels inside the dots that carry electron current are shown.

The Coulomb blockade effects in electron transport through a single dot can be taken into account in the most simple way by using the "classical" rate equations [9 12]. These equations describe the current through the dot in terms of balance between incoming and outgoing rates from (to) the leads. Basically the same classical approach has been used to calculate the conductance of coupled multi-dot systems [13, 14]. In these works the whole array of quantum dots has been treated as a single quantum system, where its many-body eigenstates were calculated by exact (numerical) diagonalization of the Hubbard Hamiltonian. Then the leads (reservoirs) were incorporated through the rate equations for single dots [11]. Yet, such a procedure is correct only for small coupling with the leads. If the 
corresponding rates $\Gamma$ are of the order of the interdot transitions $\Omega$ (Fig. 1), they affect the diagonalization of the Hubbard Hamiltonian [6]. Besides, the approach [13, 14] is mainly numerical and the inelastic scattering within the array is accounted for phenomenologically.

A different way to treat the quantum transport in multi-dot systems is to use the Blochtype rate equations [15 18] instead of the classical rate equations. In contrast with previous treatments [13,14 this approach is valid also for strong coupling with the leads and it accounts the inelastic scattering effects inside the multi-dot system [17]. It proves to be much simpler than the other approaches, so that in many cases the analytical treatment of the problem is possible [16,17]. It is also important to note that this approach is not a phenomenological one: new generalized Bloch-type rate equations were derived from the many-body Schrödinger equation with the Hubbard Hamiltonian by integrating out the continuum states of the reservoirs [17].

An important advantage of the Bloch-type equations is a clear distinction between coherent("quantum") and non-coherent ("classical") terms. The quantum coherence in the Bloch equations manifests itself in the non-diagonal density-matrix elements ("coherences"), coupled with the diagonal density-matrix elements ("probabilities") [19]. Such a coupling always takes place, whenever a carrier jumps between isolated states inside the system [17]. Otherwise, the diagonal and non-diagonal matrix elements are decoupled and the Bloch equations turn into the classical rate equation.

Actually Bloch equations describe quantum motion between isolated (non-orthogonal) states, which are directly coupled (as in the double-well potential). In general, these states can be separated by a medium with high density of states. An example of such a system is shown in Fig. 2, where two dots with aligned levels are separated by the ballistic channel. In this case a carrier transport from the left to the right reservoir proceeds through continuum states in the channel. Usually transitions from discrete to continuum states lead to dephasing, i.e. to destruction of quantum coherence. Yet, some coherent effects may survive. It is therefore important to establish of whether quantum coherence still affects the transport through separated dots, or more precisely: are the diagonal density-matrix elements still 
coupled with the non-diagonal matrix elements in the corresponding rate equations?

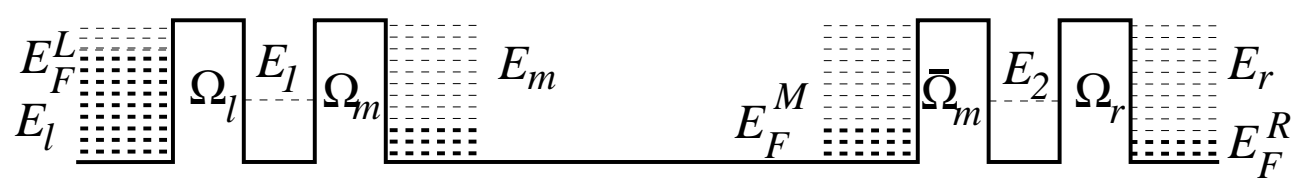

Fig. 2. Resonant transport through two quantum dots separated by a ballistic channel. Here $\Omega_{l}$ and $\Omega_{r}$ denote the coupling of the left and right dots dot with the levels $E_{l}$ and $E_{r}$ in the left and in the right leads. $\Omega_{m}$ and $\bar{\Omega}_{m}$ denote the coupling of the left and the right dots with the level $E_{m}$ in the ballistic channel.

This question leads us to a more general problem, of how to modify the Bloch rate equations for transport in coupled-dots for a general case of arbitrary distributed quantum dots. Similar to the Bloch equations one can anticipate that that the new rate equations would incorporate the classical and quantum descriptions in a very efficient way. Therefore these equations can provide most useful account of quantum coherence effects in different processes in a comparison with usual approaches [20].

In this paper we study the above mentioned problems taking as a basis the microscopic many-body Schrödinger equation with the Hubbard-type tunneling Hamiltonian, describing the entire system of the reservoirs and the quantum dots. The plan of the paper is the following. In Sect. 2 we describe the previously derived Bloch-type rate equations for coupled dots and some particular features of the coherent transport in these systems. In Sect. 3 we present the rate equations for separated dots and outline their derivation from the microscopic Schrödinger equation. The details of the derivation are described in Appendix. A comparison of coherent effects in quantum transport through coupled and separated dots is given in Sect. 4. The quantum rate equations for a general configuration of quantum dots are presented in Sect. 5. The last section is a summary. 


\section{RATE EQUATIONS AND COHERENT EFFECTS IN COUPLED QUANTUM-DOTS}

We start with a review of the quantum rate equations for coupled multi-dot systems, connected with two reservoirs (the "emitter" and the "collector") and interacting with phonon reservoir. The entire system is described by the Hubbard-type tunneling Hamiltonian where the electron-electron interaction is taken into account by introducing the corresponding electrostatic charging energy. In case of large voltage bias one can reduce the many-body Schrödinger equation to the system of quantum rate equations by integrating out continuum reservoir states [17]. As a result the following Bloch-type equations for the density matrix of the multi-dot system $\left\{\sigma_{\alpha \beta}\right\}$ are obtained, where $\alpha, \beta$ denote the isolated (non-orthogonal) states of the system:

$$
\begin{aligned}
& \dot{\sigma}_{\alpha \alpha}=i \sum_{\beta} \Omega_{\alpha \rightarrow \beta}\left(\sigma_{\alpha \beta}-\sigma_{\beta \alpha}\right)-\sigma_{\alpha \alpha} \sum_{\gamma} \Gamma_{\alpha \rightarrow \gamma}+\sum_{\delta} \sigma_{\delta \delta} \Gamma_{\delta \rightarrow \alpha}, \\
& \dot{\sigma}_{\alpha \beta}=i\left(E_{\beta}-E_{\alpha}\right) \sigma_{\alpha \beta}+i\left(\sum_{\gamma} \sigma_{\alpha \gamma} \Omega_{\gamma \rightarrow \beta}-\sum_{\delta} \Omega_{\alpha \rightarrow \delta} \sigma_{\delta \beta}\right) \\
& \quad-\frac{1}{2} \sigma_{\alpha \beta} \sum_{\delta}\left(\Gamma_{\alpha \rightarrow \delta}+\Gamma_{\beta \rightarrow \delta}\right)+\sum_{\gamma \delta \neq \alpha \beta} \sigma_{\gamma \delta} \Gamma_{\gamma \delta \rightarrow \alpha \beta} .
\end{aligned}
$$

Here $\Omega_{\alpha \rightarrow \beta}$ is the amplitude of one-electron hopping that results in the transition between

the states $\alpha$ and $\beta$. The width $\Gamma_{\alpha \rightarrow \gamma}=2 \pi \rho\left|\Omega_{\alpha \rightarrow \gamma}\right|^{2}$ is the probability per unit time for the system to make a transition from the state $|\alpha\rangle$ to the state $|\gamma\rangle$ of the device due to the tunneling to (or from) the reservoirs, or due to interaction with the phonon bath, or any other interaction generated by a continuum state medium with the density of states $\rho$. Notice that Eq. (2.1a) for diagonal elements has a form of classical rate equations, except for the first term. This term is generated by an electron hopping between the isolated levels, which results in the coupling with non-diagonal density-matrix elements. Therefore it is responsible for coherent quantum effects in electron transport. The nondiagonal matrix elements are described by Eq. (2.1b). The last term in Eq. (2.1b) appears only for systems with the number of isolated states participating in the transport is more than two. It describes the 
simultaneous conversion of the states $\gamma \rightarrow \alpha$ and $\delta \rightarrow \beta$, generated by the same one-electron decay to (from) continuum.

The current flowing through the system is given by

$$
I(t)=\sum_{\gamma} \sigma_{\gamma \gamma}(t) \Gamma_{R}^{(\gamma)}
$$

where the sum is extended over states $|\gamma\rangle$ in which the dot adjacent to the collector is occupied (we consider the electron charge $e=1$ ). $\Gamma_{R}^{(\gamma)}$ is the partial width of the state $|\gamma\rangle$ due to tunneling to the collector (the right reservoir).

It follows from Eqs. (2.1) that the coherent effects do appear in the quantum transport whenever a carrier jumps from one to another isolated state inside the device. In the absence of such transition as, for instance, in resonant tunneling through a single dot, the diagonal and non-diagonal matrix elements are decoupled and the evolution of diagonal density-matrix elements is described by the classical rate equation.

For an example we consider the quantum transport through a double-dot system at zero temperature [21], shown in Fig. 1. In order to diminish the number of equations we assume that the Coulomb repulsion does not allow for two electrons to occupy the same dot, i.e. $E_{i}+U_{i i} \gg E_{F}^{L}$, where $i=1,2$ and $U_{i i}$ is the corresponding charging energy. Yet, the interdot Coulomb repulsion $U_{12}$ is much smaller, so the both levels $E_{1,2}$ can be occupied simultaneously. In this case there are four available states of the double-dot system (for simplicity we neglected the spin): $|a\rangle$ - the levels $E_{1,2}$ are empty, $|b\rangle$ - the level $E_{1}$ is occupied, $|c\rangle$ - the level $E_{2}$ is occupied, $|d\rangle$ - the both level $E_{1,2}$ are occupied. Using Eqs. (2.1) with $\alpha, \beta, \ldots=\{a, b, c, d\}$ we find the following equations describing the time evolution of the corresponding density-matrix elements

$$
\begin{aligned}
& \dot{\sigma}_{a a}=-\Gamma_{L} \sigma_{a a}+\Gamma_{R} \sigma_{c c}, \\
& \dot{\sigma}_{b b}=\Gamma_{L} \sigma_{a a}+\Gamma_{R}^{\prime} \sigma_{d d}+i \Omega_{0}\left(\sigma_{b c}-\sigma_{c b}\right), \\
& \dot{\sigma}_{c c}=-\Gamma_{R} \sigma_{c c}-\Gamma_{L}^{\prime} \sigma_{c c}-i \Omega_{0}\left(\sigma_{b c}-\sigma_{c b}\right), \\
& \dot{\sigma}_{d d}=-\Gamma_{R}^{\prime} \sigma_{d d}+\Gamma_{L}^{\prime} \sigma_{c c},
\end{aligned}
$$




$$
\dot{\sigma}_{b c}=i\left(E_{2}-E_{1}\right) \sigma_{b c}+i \Omega_{0}\left(\sigma_{b b}-\sigma_{c c}\right)-\frac{1}{2}\left(\Gamma_{L}^{\prime}+\Gamma_{R}\right) \sigma_{b c}
$$

which are supplemented with the probability conservation condition: $\sum_{i} \sigma_{i i}(t)=1$. Note that the interdot Coulomb repulsion results in a variation of the corresponding transition rate whenever both levels are occupied $\left(\Gamma_{L, R}\right.$ is replaced by $\Gamma_{L, R}^{\prime}$, evaluated at the energy $E_{1,2}+U_{12}$ respectively [17]).

The dc current is $I=\Gamma_{R} \sigma_{c c}(t \rightarrow \infty)+\Gamma_{R}^{\prime} \sigma_{d d}(t \rightarrow \infty)$, Eq. 2.2). In order to shorten the final expression we assume that the rates are weakly dependent on the energy, $\Gamma_{L, R}^{\prime}=\Gamma_{L, R}$. Then solving Eqs. (2.3) in the limit $t \rightarrow \infty$ we obtain

$$
I=\left(\frac{\Gamma_{L} \Gamma_{R}}{\Gamma_{L}+\Gamma_{R}}\right) \frac{\Omega_{0}^{2}}{\epsilon^{2} \Gamma_{L} \Gamma_{R} /\left(\Gamma_{L}+\Gamma_{R}\right)^{2}+\Omega_{0}^{2}+\Gamma_{L} \Gamma_{R} / 4},
$$

where $\epsilon=E_{1}-E_{2}$.

It is interesting to compare Eqs. (2.3) with the corresponding classical rate equations where only transitions between the diagonal density-matrix elements take place. In this case the probabilities $\sigma_{b b}$ and $\sigma_{c c}$ of finding an electron in the first and the second dot are coupled by the some rate $\Gamma_{M}$. One easily finds that Eqs. (2.3) become

$$
\begin{aligned}
& \dot{\sigma}_{a a}=-\Gamma_{L} \sigma_{a a}+\Gamma_{R} \sigma_{c c}, \\
& \dot{\sigma}_{b b}=\Gamma_{L} \sigma_{a a}+\Gamma_{R} \sigma_{d d}-\Gamma_{M}\left(\sigma_{b b}-\sigma_{c c}\right), \\
& \dot{\sigma}_{c c}=-\Gamma_{R} \sigma_{c c}-\Gamma_{L} \sigma_{c c}+\Gamma_{M}\left(\sigma_{b b}-\sigma_{c c}\right), \\
& \dot{\sigma}_{d d}=-\Gamma_{R} \sigma_{d d}+\Gamma_{L} \sigma_{c c} .
\end{aligned}
$$

(Cf. with the analogues rate equations in [22,23]). Solving these classical rate equations in the limit $t \rightarrow \infty$ we obtain for the (classical) dc current

$$
I_{c l}=\frac{\Gamma_{L} \Gamma_{R} \Gamma_{M}}{\Gamma_{L} \Gamma_{R}+\Gamma_{L} \Gamma_{M}+\Gamma_{R} \Gamma_{M}}
$$

Comparing Eq. (2.6) with its "quantum" counterpart, Eq. (2.4), we find that the quantum-mechanical nature of the dc current displays itself in the Lorentzian-shape resonance as a function of the levels disalignment, $\epsilon=E_{2}-E_{1}$. Such resonances were already 
observed in the coupled-dot systems [3]. Yet, the existence of the resonance is not necessarily related to the quantum coherence effect. The later manifests itself in a peculiar and even a counter-intuitive dependence of the resonant current on coupling with the reservoirs. It follows from Eq. (2.4) that the current decreases when the coupling with the reservoirs increases. For instance, the peak value of the resonant current $I_{\epsilon=0} \propto \Gamma_{L} / \Gamma_{R} \rightarrow 0$ for $\Gamma_{R} \gg \Gamma_{L}$. It implies that by increasing the penetrability of the barrier, connecting the second dot with the collector we diminish the total current. As an example we show in Fig. 3 the peak value of the resonant current as a function of $\Gamma_{R}$ for constant $\Gamma_{L}$ and $\Omega_{0}$ (the solid line). This effect can be observed in coupled-dot structures by changing the corresponding gate voltage.

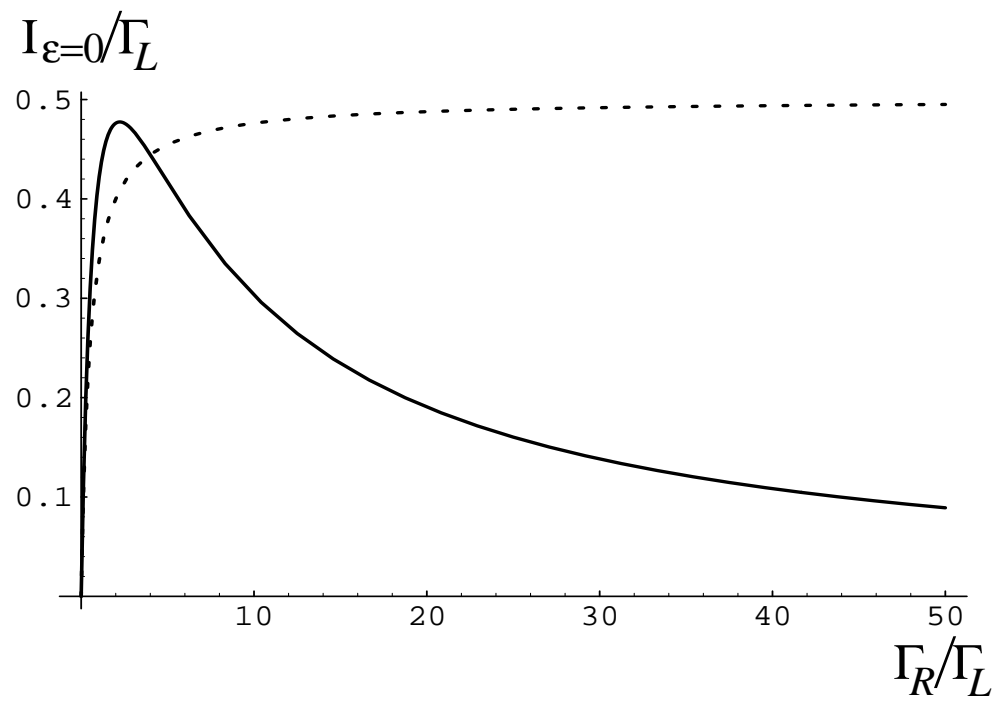

Fig. 3. Current through the coupled-dot structure, Fig. 1, as a function of $\Gamma_{R} / \Gamma_{L}$. The solid line corresponds to Eq. (2.4) for $\epsilon=0$ and $\Gamma_{L}^{2} / 4 \Omega_{0}^{2}=5$. The dashed line is the result of the classical rate equations, Eq. (2.6), for $\Gamma_{M}=\Gamma_{L}$

For a comparison the corresponding "classical" current $I_{c l}$, Eq. (2.6), is shown in Fig. 3 
by the dashed line.

The decrease of the resonant current shown in Fig. 3 is a result of destructive quantum interference during decay of a coherent superposition to continuum in the regime of strong coupling with the reservoirs [23,24]. A similar effect had been observed in electron decay from coupled quantum wells to continuum [25]. It is quite interesting that such a counterintuitive behavior of the resonant current looks as a manifestation of the Zeno effect [26]. It tells us that the quantum transitions between different states slow down when one of the states is continuously observed. Indeed, the increase of the right barrier penetrability leads to immediate tunneling of an electron to the collector whenever it arrives to the second dot. It can be considered as a continuous observation of the second dot state, which results in an effective electron localization in the first dot [27].

\section{RATE EQUATIONS FOR TWO SEPARATED DOTS}

Now we are going to derive rate equations for quantum transport in mesoscopic systems with arbitrary configuration of quantum dots. As a generic example we consider quantum transport through two quantum dots separated by a ballistic channel, Fig. 2. The dots contain only isolated levels, whereas the density of states in the ballistic channel and in the emitter and the detector is very high (continuum). This system can be described by the tunneling Hamiltonian

$$
\begin{aligned}
\mathcal{H} & =\sum_{l} E_{l} a_{l}^{\dagger} a_{l}+E_{1} a_{1}^{\dagger} a_{1}+\sum_{m} E_{m} a_{m}^{\dagger} a_{m}+E_{2} a_{2}^{\dagger} a_{2} \sum_{r} a_{r} a_{r}^{\dagger} a_{r}+\sum_{i, j=1,2} U_{i j} n_{i} n_{j} \\
& +\left\{\sum_{l} \Omega_{l} a_{1}^{\dagger} a_{l}+\sum_{m} \Omega_{m} a_{m}^{\dagger} a_{1}+\sum_{m} \bar{\Omega}_{m} a_{2}^{\dagger} a_{m}+\sum_{r} \Omega_{r} a_{r}^{\dagger} a_{2}+\text { H.c. }\right\}
\end{aligned}
$$

Here the subscripts $l, m$ and $r$ enumerate correspondingly the levels in the left reservoir, in the (middle) ballistic channel and in the right reservoir. The spin degrees of freedom were omitted. In order to simplify the derivation we assumed that the intradot charging energy $U_{i i}$ is large, $E_{1,2}+U_{i i} \gg E_{F}^{L}$. Thus only one electron can occupy each of the dots. However, the interdot charging energy $U_{12}$ is much smaller, so it does not prevent simultaneous occupation 
of the two dots. The same is assumed for the Coulomb repulsion between electrons inside the dots and the ballistic channel. Although we did not include this interaction in the Hamiltonian (3.1), it can be treated in the same way as the interdot interaction $U_{12}$. As in the previous case we restrict ourselves to the zero temperature case, even though the results are valid for a finite temperature, as would be clear from the derivation.

Let us assume that all the levels in the emitter, in the ballistic channel and in the collector are initially filled up to the Fermi energies $E_{F}^{L}, E_{F}^{M}$ and $E_{F}^{R}$ respectively. We call it as the "vacuum" state, $|0\rangle$. (In the following we consider the case of large bias, so that $\left.E_{F}^{L} \gg E_{F}^{M}, E_{F}^{R}\right)$. This vacuum state is unstable: the Hamiltonian Eq. (3.1) requires it to decay exponentially to continuum states having the form $a_{1}^{\dagger} a_{l}|0\rangle$ with an electron in the level $E_{1}$ and a hole in the emitter continuum, $a_{m}^{\dagger} a_{l}|0\rangle$ with an electron in the level $E_{m}$ in the ballistic channel and a hole in the emitter, and so on. The many-body wave function describing this system can be written in the occupation number representation as

$$
\begin{aligned}
|\Psi(t)\rangle & =\left[b_{0}(t)+\sum_{l} b_{1 l}(t) a_{1}^{\dagger} a_{l}+\sum_{l, m} b_{l m}(t) a_{m}^{\dagger} a_{l}+\sum_{l} b_{2 l}(t) a_{2}^{\dagger} a_{l}\right. \\
& \left.+\sum_{l, r} b_{l r}(t) a_{r}^{\dagger} a_{l}+\sum_{l<l^{\prime}} b_{12 l l^{\prime}}(t) a_{1}^{\dagger} a_{2}^{\dagger} a_{l} a_{l^{\prime}}+\sum_{l<l^{\prime}, r} b_{1 l l^{\prime} r}(t) a_{1}^{\dagger} a_{r}^{\dagger} a_{l} a_{l^{\prime}}+\ldots\right]|0\rangle,
\end{aligned}
$$

where $b(t)$ are the time-dependent probability amplitudes to find the system in the corresponding states described above. These amplitudes are obtained from the Shrödinger equation $i|\dot{\Psi}(t)\rangle=\mathcal{H}|\Psi(t)\rangle$, supplemented with the initial condition $\left(b_{0}(0)=1\right.$, and all the other $b(0)$ 's being zeros). Using the amplitudes $b(t)$ we can find the density-matrix of the quantum dots, $\sigma_{i j}^{(k, n)}(t)$, by tracing out the continuum states of the reservoirs and the ballistic channel. Here the subscript indices in $\sigma$ denote four states of the dots: $i, j=\{a, b, c, d\}$, where $|a\rangle$ - the levels $E_{1,2}$ are empty, $|b\rangle$ - the level $E_{1}$ is occupied, $|c\rangle$ - the level $E_{2}$ is occupied, $|d\rangle$ - the both level $E_{1,2}$ are occupied, and the superscript indices $k, n$ denote the number of electrons accumulated in the ballistic channel and in the collector respectively at time $t$, Fig. 4. 


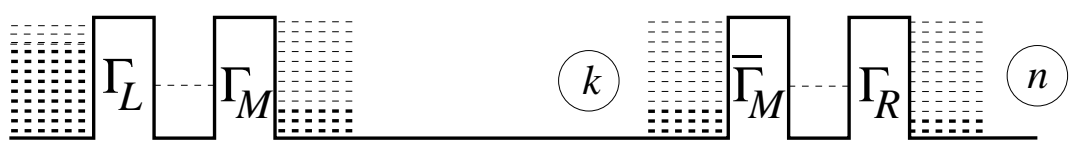

(a)

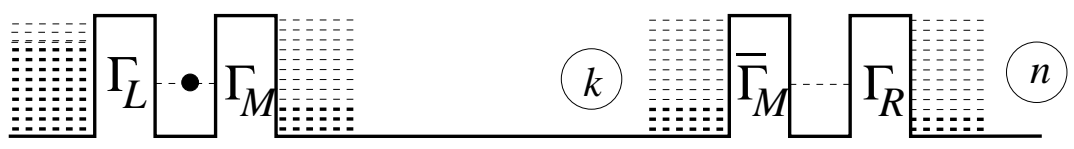

(b)

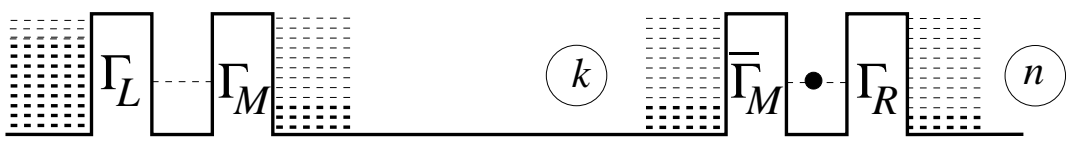

(c)

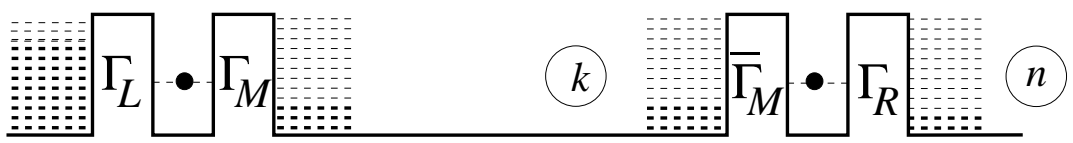

(d)

Fig. 4. Electron states of the two separated dot structure, shown in Fig. 2. $\Gamma_{L, R}, \Gamma_{M}$ and $\bar{\Gamma}_{M}$ are the tunneling rates between the dots and the reservoirs, and between the dots and the ballistic channel. The indices $k$ and $n$ denote the number of electrons penetrating to the ballistic channel and to the collector at time $t$.

One finds

$$
\begin{aligned}
& \sigma_{a a}^{(0,0)}(t)=\left|b_{0}(t)\right|^{2}, \quad \sigma_{a a}^{(1,0)}(t)=\sum_{l, m}\left|b_{l m}(t)\right|^{2}, \quad \sigma_{a a}^{(1,1)}(t)=\sum_{l<l^{\prime}, m, r}\left|b_{l l^{\prime} m r}(t)\right|^{2}, \quad \ldots \\
& \sigma_{b b}^{(0,0)}(t)=\sum_{l}\left|b_{1 l}(t)\right|^{2}, \quad \sigma_{b b}^{(1,0)}(t)=\sum_{l<l^{\prime}, m}\left|b_{1 l l^{\prime} m}(t)\right|^{2}, \\
& \ldots \ldots \ldots \ldots \\
& \ldots \ldots \ldots \\
& \ldots \ldots \\
& \sigma_{b c}^{(1,1)}(t)=\sum_{l<l^{\prime}<l^{\prime \prime}, m, r}\left|b_{1 l l^{\prime} l^{\prime \prime} m r}(t)\right|^{2}, \quad \ldots \\
& \sigma_{b c}^{(1,1)}(t)=\sum_{l} b_{1 l}(t) b_{2 l}^{*}(t), \quad \sigma_{b c}^{(1,0)}(t)=\sum_{l<l^{\prime}<l^{\prime \prime}, m, r} b_{1 l l^{\prime} m}(t) b_{2 l l^{\prime} m}^{*}(t),
\end{aligned}
$$

The rate of electrons arriving to the collector determines the electron current in the 
system. Therefore the current operator is $\hat{I}=i\left[\mathcal{H}, \hat{N}_{R}\right]$, where $\hat{N}_{R}=\sum_{r} a_{r}^{\dagger} a_{r}$ is the operator for the total number of electrons accumulated in the right reservoir. Using Eqs. (3.1), (3.2), (3.3) we find that the current $I(t)$ flowing through the system is

$$
I(t)=\langle\Psi(t)|\hat{I}| \Psi(t)\rangle=\sum_{k, n} n\left[\dot{\sigma}_{a a}^{(k, n)}(t)+\dot{\sigma}_{b b}^{(k, n)}(t)+\dot{\sigma}_{c c}^{(k, n)}(t)+\dot{\sigma}_{d d}^{(k, n)}(t)\right]
$$

As expected, $I(t)$ is the time derivative of the total charge accumulated in the collector.

It follows from Eq. (3.4) that the current $I(t)$ flowing through this system is expressed in terms of the diagonal elements of the density-matrix $\sigma(t)$. In order find the differential equations for $\sigma(t)$ we need to sum over the states of the reservoirs and the ballistic channel, Eqs. (3.3). It can be done analytically by using the procedure similar to that in Refs. [17,18], providing that the levels are not close to Fermi levels, $\left|E_{i}-E_{F}\right| \gg \Gamma$, Fig. 4. The details of derivation are described in Appendix. As a result we obtain the following Bloch-type rate equations for the matrix elements of the density-submatrix $\sigma(t)$ :

$$
\begin{aligned}
& \dot{\sigma}_{a a}^{(k, n)}=-\Gamma_{L} \sigma_{a a}^{(k, n)}+\Gamma_{M} \sigma_{b b}^{(k-1, n)}+\bar{\Gamma}_{M} \sigma_{c c}^{(k-1, n)}+\Gamma_{R} \sigma_{c c}^{(k, n-1)} \\
& +2 \pi \rho_{M} \Omega_{M} \bar{\Omega}_{M}\left(\sigma_{b c}^{(k-1, n)}+\sigma_{c b}^{(k-1, n)}\right) \\
& \dot{\sigma}_{b b}^{(k, n)}=-\Gamma_{M} \sigma_{b b}^{(k, n)}+\Gamma_{L} \sigma_{a a}^{(k, n)}+\bar{\Gamma}_{M} \sigma_{d d}^{(k-1, n)}+\Gamma_{R} \sigma_{d d}^{(k, n-1)} \\
& -\pi \rho_{M} \Omega_{M} \bar{\Omega}_{M}\left(\sigma_{b c}^{(k, n)}+\sigma_{c b}^{(k, n)}\right) \\
& \dot{\sigma}_{c c}^{(k, n)}=-\left(\Gamma_{L}+\bar{\Gamma}_{M}+\Gamma_{R}\right) \sigma_{c c}^{(k, n)}+\Gamma_{M} \sigma_{d d}^{(k-1, n)}-\pi \rho_{M} \Omega_{M} \bar{\Omega}_{M}\left(\sigma_{b c}^{(k, n)}+\sigma_{c b}^{(k, n)}\right) \\
& \dot{\sigma}_{d d}^{(k, n)}=-\left(\Gamma_{M}+\bar{\Gamma}_{M}+\Gamma_{R}\right) \sigma_{d d}^{(k, n)}+\Gamma_{L} \sigma_{c c}^{(k, n)} \\
& \dot{\sigma}_{b c}^{(k, n)}=i\left(E_{2}-E_{1}\right) \sigma_{b c}^{(k, n)}-\pi \rho_{M} \Omega_{M} \bar{\Omega}_{M}\left(\sigma_{b b}^{(k, n)}+\sigma_{c c}^{(k, n)}\right)+2 \pi \rho_{M} \Omega_{M} \bar{\Omega}_{M} \sigma_{d d}^{(k-1, n)} \\
& -\frac{1}{2}\left(\Gamma_{L}+\Gamma_{M}+\bar{\Gamma}_{M}+\Gamma_{R}\right) \sigma_{b c}^{(k, n)} .
\end{aligned}
$$

Here $\Gamma$ denote the tunneling widths to the leads and the ballistic channel $\left(\Gamma=2 \pi \rho|\Omega|^{2}\right.$, where $\rho$ is the corresponding density of states), and $\Omega_{M}, \bar{\Omega}_{M}$ are the hopping amplitudes between the left and the right dots and the states $E_{m}=E_{1,2}$ of the ballistic channel.

Equations (3.5) have clear physical interpretation. Consider for instance Eq. (3.5a) for the probability rate of finding the system in the state $a$ with $k$ electrons in the ballistic 
channel and $n$ electrons in the right reservoir (Fig. 4a). This state decays with the rate $\Gamma_{L}$ into the state $b$ (Fig. 4b) whenever an electron enters the first dot from the left reservoir. This process is described by the first term in Eq. (3.5a). On the other hand, the states $b$ and $c$ (Figs. 4b, 4c) with $k-1$ electrons in the ballistic channel decay into the state $a$ with $k$ electrons in the ballistic channel. It takes place due to one-electron tunneling from the quantum dots into the ballistic channel with the rates $\Gamma_{M}$ and $\bar{\Gamma}_{M}$ respectively. This process is described by the second and the third terms in Eq. (3.5a). Also the state $c$ (Fig. 4c) with $n-1$ electrons in the right reservoir can decay into the state $a$ due to tunneling to the right reservoir with the rate $\Gamma_{R}$ (the fourth term in Eq. (3.5a)). The last term in this equation describes the decay of coherent superposition of the states $b$ and $c$ into the state a. It takes place due to single electron tunneling from the first and the second dots into the same state of the ballistic channel with the amplitudes $\Omega_{M}$ and $\bar{\Omega}_{M}$, respectively. Obviously, this process has no classical analogy, since classical particle cannot simultaneously occupy two dots.

Equations (3.5b), (3.5c) and (3.5d) describe the probability rate of finding the system in the states where one of the dots or both dots are occupied. In the first case an electron can jump into unoccupied dot via continuum states of the ballistic channel. As a result, the states $b$ and $c$ can decay into linear superposition of the states $b$ and $c$. This process is described by the last terms in Eqs. (3.5b), (3.5d). Obviously, if the both dots are occupied, such a process cannot take a place. Therefore $\sigma_{d d}$ is not coupled with the nondiagonal densitymatrix elements, Eq. (3.5d). The last equation, (3.5e) describes the time-dependence of the nondiagonal density matrix element. It has the same interpretation as all previous equations.

Equations (3.5) for the reduced density matrix $\sigma(t)$ were derived starting from the wave function $|\Psi(t)\rangle$, Eq. (3.2), instead of using the density matrix for the entire system. Of course, it makes no difference if the entire system is initially in the pure state, or all the levels of the reservoirs are occupied up to the corresponding Fermi energies. At finite temperature, however, the system is not initially in a pure state. Then one needs to perform the derivation using the Liouville (Landau-von Neumann) equation for the density-matrix. Yet, if energy 
levels $E_{1,2}$ of the dots are far away from the Fermi-levels $\left(T \ll E_{F}^{L}-E_{1,2}, E_{1,2}-E_{F}^{R}\right)$, then the reservoir levels that carry the current $\left(\left|E_{l}-E_{1,2}\right| \lesssim \Gamma\right)$ are deeply inside the Fermi sea. In this case one can consider these levels as fully occupied. Then neglecting the relaxation processes in the reservoir and assuming zero temperature inside the dots, we would arrive to the same Eqs. (3.5), derived from the pure state.

Using Eqs. (3.5) one finds for the total current, Eq. (3.4)

$$
I(t)=\Gamma_{R}\left[\sigma_{c c}(t)+\sigma_{d d}(t)\right]
$$

where $\sigma_{i i}=\sum_{k, n} \sigma_{i i}^{(k, n)}$ are the total "probabilities". We can easily understand this result by taken into account that $\sigma_{c c}+\sigma_{d d}$ is the total probability for occupation of the second dot and $\Gamma_{R}$ is the rate of electron transitions from this dot to the (adjacent) right reservoir, Eq. (2.2).

In order to find differential equations for $\sigma_{i j}$ we sum over $k, n$ in Eqs. (3.5). Then we obtain the following Bloch-type equations, which describe the time-dependence of the density-matrix for separated dots

$$
\begin{aligned}
& \dot{\sigma}_{a a}=-\Gamma_{L} \sigma_{a a}+\Gamma_{M} \sigma_{b b}+\bar{\Gamma}_{M} \sigma_{c c}+\Gamma_{R} \sigma_{c c}+2 \pi \rho_{M} \Omega_{M} \bar{\Omega}_{M}\left(\sigma_{b c}+\sigma_{c b}\right) \\
& \dot{\sigma}_{b b}=-\Gamma_{M} \sigma_{b b}+\Gamma_{L} \sigma_{a a}+\bar{\Gamma}_{M} \sigma_{d d}+\Gamma_{R} \sigma_{d d}-\pi \rho_{M} \Omega_{M} \bar{\Omega}_{M}\left(\sigma_{b c}+\sigma_{c b}\right) \\
& \dot{\sigma}_{c c}=-\left(\Gamma_{L}+\bar{\Gamma}_{M}+\Gamma_{R}\right) \sigma_{c c}+\Gamma_{M} \sigma_{d d}-\pi \rho_{M} \Omega_{M} \bar{\Omega}_{M}\left(\sigma_{b c}+\sigma_{c b}\right) \\
& \dot{\sigma}_{d d}=-\left(\Gamma_{M}+\bar{\Gamma}_{M}+\Gamma_{R}\right) \sigma_{d d}+\Gamma_{L} \sigma_{c c} \\
& \dot{\sigma}_{b c}=i\left(E_{2}-E_{1}\right) \sigma_{b c}-\pi \rho_{M} \Omega_{M} \bar{\Omega}_{M}\left(\sigma_{b b}+\sigma_{c c}\right)+2 \pi \rho_{M} \Omega_{M} \bar{\Omega}_{M} \sigma_{d d}-\frac{1}{2} \Gamma_{t o t} \sigma_{b c},
\end{aligned}
$$

where $\Gamma_{t o t}=\Gamma_{L}+\Gamma_{M}+\bar{\Gamma}_{M}+\Gamma_{R}$.

The stationary (dc) current $I=I(t \rightarrow \infty)$ Eq. (3.6) can be easily obtained from Eqs. (3.7) by taken into account that $\dot{\sigma}_{i j} \rightarrow 0$ for $t \rightarrow \infty$. Then Eqs. (3.7) turn into a system of linear algebraic equations, supplemented by a probability conservation condition $\sigma_{a a}+\sigma_{b b}+\sigma_{c c}+$ $\sigma_{d d}=1$. Consider, for example, the case of the same partial widths of the levels $E_{1,2}$, i.e. $\Gamma_{L}=\Gamma_{R}=\Gamma_{0}$ and $\Gamma_{M}=\bar{\Gamma}_{M}$. The latter implies $2 \pi \rho_{M} \Omega_{M} \bar{\Omega}_{M}= \pm \Gamma_{M}$, since the amplitudes $\Omega_{M}, \bar{\Omega}_{M}$ can be of the opposite signs. Solving Eqs. (3.6), (3.7) one finds for dc current 


$$
I=\frac{\Gamma_{0}^{2} \Gamma_{M}^{2}}{2 \epsilon^{2}\left(\Gamma_{0}+\Gamma_{M}\right)+2 \Gamma_{0}\left(\Gamma_{0}^{2}+3 \Gamma_{0} \Gamma_{M}+3 \Gamma_{M}^{2}\right)},
$$

where $\epsilon=E_{1}-E_{2}$.

Similar to the coupled-dot case, Eq. (2.4), dc current in separated dots displays the Lorentzian shape resonance as a function of $\epsilon$ and the same peculiar dependence on the coupling with the leads. The latter manifests the quantum-coherence effects in separated dot systems. We discuss these effects in the next section.

\section{COHERENT EFFECTS IN TWO-DOT SYSTEMS}

It follows from the rate equations (3.7) that the diagonal density-matrix elements are coupled with the non-diagonal terms $\left(\sigma_{b c}, \sigma_{c b}\right)$, similar to Eqs. (2.3) for a coupled-dot system. It means that the coherent effects should survive even in separated quantum dots. Indeed, let us consider the dependence of the resonant current on a coupling with the reservoirs. As an example, we show in Fig. 5a the peak value of the resonant current $(\epsilon=0)$ obtained from Eqs. (3.6), (3.7) as a function of $\Gamma_{R}$ at fixed $\Gamma_{L}=\Gamma_{M}$. One finds that the current through the dots separated by a ballistic channel displays the same peculiar behavior with $\Gamma_{R}$, as in the case of coupled dots, Fig. 3. Namely, the current decreases when the penetrability of the right barrier increases. Such a behavior of the resonant current is a clear manifestation of the quantum coherence effects, as explained in Sect. II (see also 23 25]). It also can be verified experimentally by changing the voltage on the gate, connecting the right dot with the collector.

On the other hand, the dependence of the resonant current on the width $\Gamma_{M}$ is quite different, Fig. 5b. One finds that the current increases with $\Gamma_{M}$. It is not surprising, since $\Gamma_{M}$ plays a role of coupling between the two dots. In fact, a similar behavior with the coupling $\Omega_{0}$ displays the resonant current in the case of coupled dots, Eq. (2.4).

Despite the analogues quantum coherence effects, the rate equations (3.7) are different from their counterpart for the coupled-dots, Eqs. (2.3). One finds that the diagonal matrix elements in Eqs. (3.7) are coupled with the real part of the non-diagonal matrix element, 


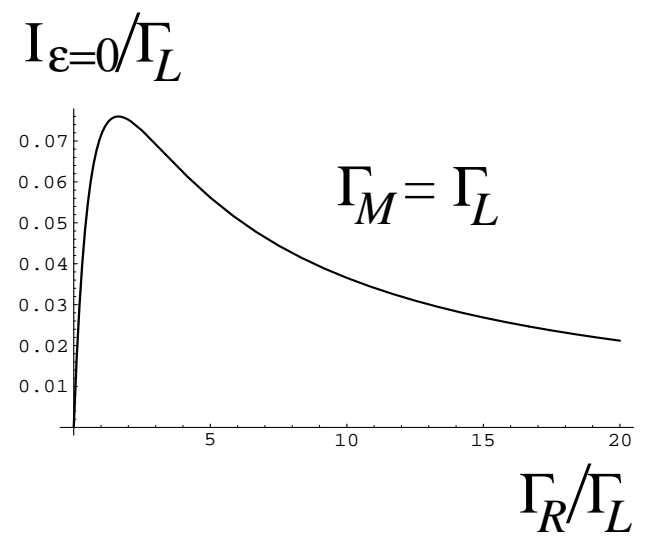

(a)

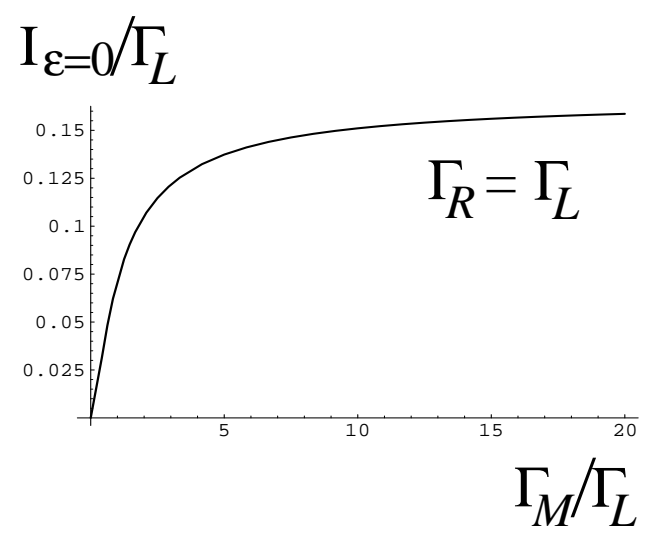

(b)

Fig. 5. DC current through the separated dot structure, Fig. 2, as a function of $\Gamma_{R} / \Gamma_{L}$ for $\Gamma_{M}=\Gamma_{L}(\mathrm{a})$, and as a function of $\Gamma_{M} / \Gamma_{L}$ for $\Gamma_{R}=\Gamma_{L}(\mathrm{~b})$.

whereas the corresponding terms in Eqs. (2.3), are coupled with the imaginary part of the non-diagonal matrix element. Notice also that the coupling is determined by the hopping amplitude $\Omega$ in Eqs. (2.3), while the corresponding coupling in Eqs. (3.7) is proportional to $\Omega^{2} \rho$

It is important to point out that the coupling with the non-diagonal density-matrix elements in Eqs. (3.7) does not decrease with the separation distance between the dots, although the hopping amplitudes $\Omega_{M}, \bar{\Omega}_{M}$ do decrease. Indeed, using semi-classical expressions one finds $\left|\Omega_{M}\right|=\left(1 / \sqrt{\tau_{1} \tau_{M}}\right) \exp (-S)$ and $\left|\bar{\Omega}_{M}\right|=\left(1 / \sqrt{\tau_{2} \tau_{M}}\right) \exp (-\bar{S})$, where $S(\bar{S})$ is the action under the barrier, separating the first (second) dot from the ballistic channel. It can be written as $S=\int_{x_{i}}^{x_{f}}|p(x)| d x$, where $|p(x)|=\sqrt{2 m[V(x)-E]}$ and $x_{i, f}$, are the classical turning points. $\tau_{1,2}$ are the classical periods of motion in the first (second) quantum well and $\tau_{M}$ is the classical periods of motion in the ballistic channel. It implies that $\tau_{M} \rightarrow \infty$ when the length of the ballistic channel increases. Yet, $\tau_{M}$ is canceled out from the coupling between the diagonal and non-diagonal matrix elements, since the ballistic channel is effectively one-dimensional so that the density of states is $\rho_{M}=\tau_{M} / 2 \pi$. Finally we obtain

$$
2 \pi \rho_{M} \Omega_{M} \bar{\Omega}_{M}= \pm \frac{1}{2 \pi \sqrt{\tau_{1} \tau_{2}}} \exp [-(S+\bar{S})]
$$


Therefore, the quantum coherence effects in dc current could survive even for long ballistic channels. Obviously, it is true only for ideal one-dimensional channels, where all the flux coming from the first dot arrives the second dot. It should be also pointed out that electronelectron scattering inside the ballistic channel (not taken into account in these calculations) may reduce the interference effects. Yet, we expect that such a process would not diminish the coupling (4.1), but increase the "dissipation" width $\Gamma_{t o t}$ in Eq. (3.78). This problem, however, needs a special investigation.

It is interesting to make a comparison between peak values of the dc current in coupled and separated dots. In both cases the current reaches its maximal value for $\Gamma_{L}=\Gamma_{R}=\Gamma_{0}$ and $\epsilon=0$, Eqs. (2.4), (3.8). One finds for the coupled dots

$$
I_{\max }=\frac{\Gamma_{0}}{2} \frac{\Omega_{0}^{2}}{\Omega_{0}^{2}+\Gamma_{0}^{2} / 4} \rightarrow \frac{\Gamma_{0}}{2}, \quad \text { for } \quad \Omega_{0} \gg \Gamma_{0},
$$

while for the separated dots

$$
I_{\max }=\frac{\Gamma_{0}}{2} \frac{\Gamma_{M}^{2}}{3 \Gamma_{M}^{2}+3 \Gamma_{0} \Gamma_{M}+\Gamma_{0}^{2}} \rightarrow \frac{\Gamma_{0}}{6}, \quad \text { for } \quad \Gamma_{M} \gg \Gamma_{0},
$$

Thus the peak value of dc current in the coupled dots is three times larger than that in the separated dots.

It is quite natural to associate quantum coherence effects in double-well systems with quantum oscillations of an electron between the wells. Indeed, an electron in a coupled-dot system, detached from the emitter and the collector, oscillates between the states (a) and (b), Fig. 6. These oscillations are reproduced by the same rate equations that describe quantum transport in coupled-dots, Eqs. (2.3), but now with $\Gamma_{L}=\Gamma_{R}=0$. We then obtain for the probability of finding an electron in the states (a) and (b), $\sigma_{a a}(t)$ and $\sigma_{b b}(t)$, Fig.6:

$$
\begin{aligned}
& \dot{\sigma}_{a a}=i \Omega_{0}\left(\sigma_{a b}-\sigma_{b a}\right) \\
& \dot{\sigma}_{b b}=-i \Omega_{0}\left(\sigma_{a b}-\sigma_{b a}\right) \\
& \dot{\sigma}_{a b}=i\left(E_{2}-E_{1}\right) \sigma_{a b}+i \Omega_{0}\left(\sigma_{a a}-\sigma_{b b}\right) .
\end{aligned}
$$


(a)

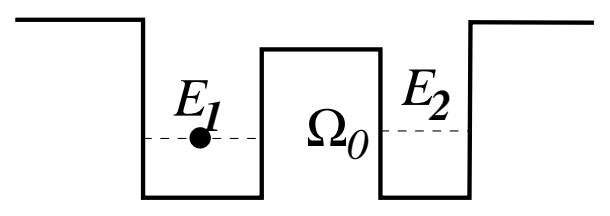

(b)

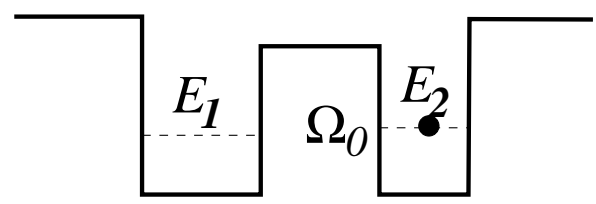

Fig. 6. Electron states of the coupled-dot structure of Fig. 1, isolated from the leads.

Solving this equations with the initial condition $\sigma_{a a}(0)=1$ and $\sigma_{b b}(0)=0$, we obtain

$$
\sigma_{a a}(t)=\frac{\Omega_{0}^{2} \cos ^{2}\left(\omega_{1} t\right)+\epsilon^{2} / 4}{\Omega_{0}^{2}+\epsilon^{2} / 4}
$$

where $\omega_{1}=(1 / 2) \sqrt{4 \Omega_{0}^{2}+\epsilon^{2}}$. As expected, an electron oscillates between the dots with an amplitude $1 /\left(1+\epsilon^{2} / 4 \Omega_{0}^{2}\right)$.

The situation is different, however, if the dots are separated by a ballistic channel, Fig. 7 . Indeed, the corresponding rate equations for the probabilities $\sigma_{a a}(t)$ and $\sigma_{b b}(t)$ of finding an electron in the states (a) and (b) of Fig. 7 are obtained from Eqs. (3.7) for $\Gamma_{L}=\Gamma_{R}=0$. Consider for simplicity the case of $\Omega_{M}=\bar{\Omega}_{M}$. Then Eqs. (3.7) become

$$
\begin{aligned}
& \dot{\sigma}_{a a}=-\Gamma_{M} \sigma_{a a}-\frac{1}{2} \Gamma_{M}\left(\sigma_{a b}+\sigma_{b a}\right) \\
& \dot{\sigma}_{b b}=-\Gamma_{M} \sigma_{b b}-\frac{1}{2} \Gamma_{M}\left(\sigma_{a b}+\sigma_{b a}\right) \\
& \dot{\sigma}_{a b}=i\left(E_{2}-E_{1}\right) \sigma_{a b}-\frac{1}{2} \Gamma_{M}\left(\sigma_{a a}+\sigma_{b b}\right)-\Gamma_{M} \sigma_{a b}
\end{aligned}
$$

Solving these equations with the initial condition $\sigma_{a a}(0)=1$ and $\sigma_{b b}(0)=0$, we obtain

$$
\sigma_{a a}(t)=\frac{\Gamma_{M}^{2} \cosh ^{2}\left(\omega_{2} t\right)-\epsilon^{2}}{\Gamma_{M}^{2}-\epsilon^{2}} \exp \left(-\Gamma_{M} t\right),
$$

where $\omega_{2}=(1 / 2) \sqrt{\Gamma_{M}^{2}-\epsilon^{2}}$. It follows from Eq (4.7) that $\sigma_{a a}(t)$ does not display any 


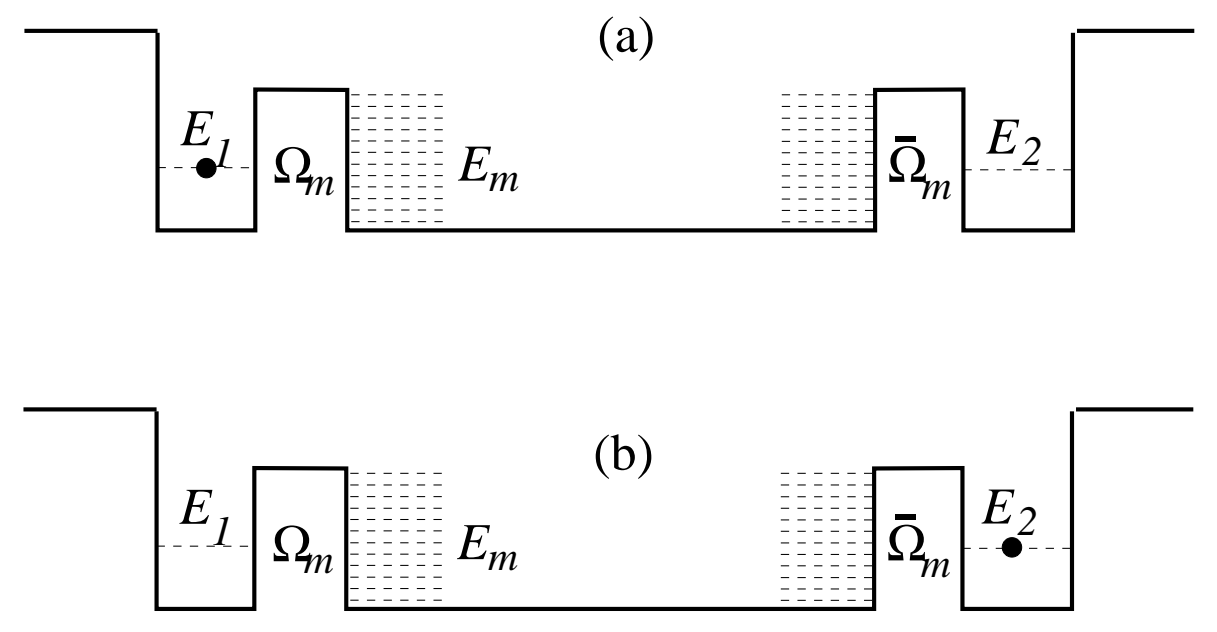

Fig. 7. Electron states of the two separate dot structure of Fig. 2, isolated from the leads.

oscillations. As an example, we show in Fig. 8 the probability of finding an electron in the left dot as a function of time in the case of coupled and separated dots (Figs. 6, 7) for $\epsilon=\Gamma_{M}=\Omega_{0}$. The solid line corresponds to Eq. (4.5), and the dashed line to Eq. (4.7). Notice that $\sigma_{a a}=\sigma_{b b} \rightarrow 1 / 4$ for $t \rightarrow \infty$ when the levels are aligned $(\epsilon=0)$, and $\sigma_{a a}=\sigma_{b b} \rightarrow 0$ for $t \rightarrow \infty$ when $\epsilon \neq 0$. It means that an electron decays into the ballistic channel.

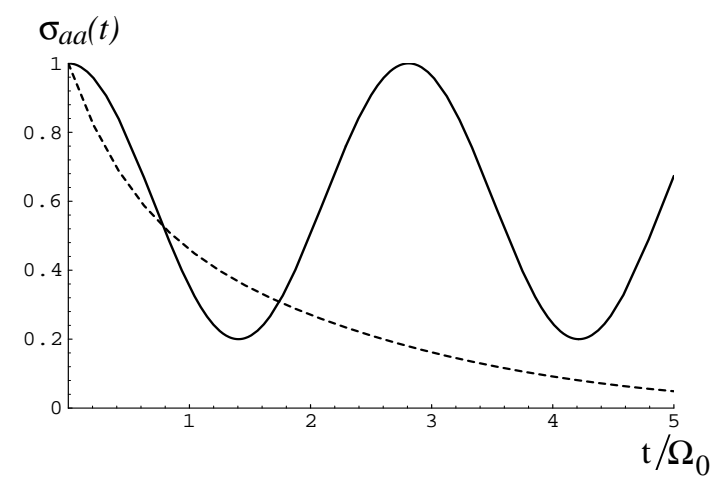

Fig. 8. The occupation of the left dot in Figs. 6 and 7 as a function of time for $\epsilon=\Gamma_{M}=\Omega_{0}$. The solid line corresponds to coupled dots, and the dashed line to separated dots. 
The reason for a dissipative behavior of $\sigma_{a a}$ in the case separated dots is a coupling of the dots with continuum states of the ballistic channel. The latter leads to dephasing that destroys quantum oscillations. It is thus rather remarkable that the quantum coherence effects would "reappear" when the same system is connected with the leads, Fig. 2.

\section{GENERAL CASE}

The rate equations (3.7), describing electron transport in separated dots can be extended to any multi-dot system. By applying the same technique of integrating out the reservoir states as in Ref. [17] and in Appendix, we arrive to the rate equations for the density-matrix $\sigma_{\alpha \beta}$ of the multi-dot system. These equations can be written as

$$
\begin{aligned}
\dot{\sigma}_{\alpha \beta} & =i\left(E_{\beta}-E_{\alpha}\right) \sigma_{\alpha \beta}+i\left(\sum_{\gamma} \sigma_{\alpha \gamma} \tilde{\Omega}_{\gamma \rightarrow \beta}-\sum_{\gamma} \tilde{\Omega}_{\alpha \rightarrow \gamma} \sigma_{\gamma \beta}\right) \\
& -\sum_{\gamma, \delta}^{\prime} \pi \rho\left(\sigma_{\alpha \gamma} \Omega_{\gamma \rightarrow \delta} \Omega_{\delta \rightarrow \beta}+\sigma_{\gamma \beta} \Omega_{\gamma \rightarrow \delta} \Omega_{\delta \rightarrow \alpha}\right)+\sum_{\gamma, \delta} \pi \rho\left(\Omega_{\gamma \rightarrow \alpha} \Omega_{\delta \rightarrow \beta}+\Omega_{\gamma \rightarrow \beta} \Omega_{\delta \rightarrow \alpha}\right) \sigma_{\gamma \delta} .
\end{aligned}
$$

Here $|\alpha\rangle,|\beta\rangle, \ldots$ are the states of the multi-dot system in the occupation number representation, and $\Omega_{\alpha \rightarrow \beta}$ denotes one-electron hopping amplitude that generates $\alpha \rightarrow \beta$-transition. We distinguish between the amplitudes $\tilde{\Omega}$ and $\Omega$ of one-electron hopping among isolated states and among isolated and continuum states, respectively. The latter transitions are of the second order in the hopping amplitude $\sim \Omega^{2}$. These transition are produced by two consecutive hoppings of an electron with the same energy across continuum states with the density of states $\rho$. The symbol $\sum^{\prime}$ means that only those transitions $\gamma \rightarrow \delta \rightarrow(\alpha, \beta)$ are accounted for in the sum, where the number of electrons in continuum states (in the reservoirs and ballistic channels) stays the same in the initial and final states (c.f. Eqs. (3.5)).

It is rather easy to verify that Eqs. (5.1) coincide with Eqs. (3.7) for $\alpha, \beta, \ldots=\{a, b, c, d\}$, which are the states of the separated dot system, shown in Fig. 5. Let us compare now Eqs. (5.1) with the Bloch rate equations (2.1) for quantum transport in coupled dots. We begin with the equations for diagonal density-matrix elements, $\beta=\alpha$. The first term in 
Eq. (5.1) is zero, since $E_{\alpha}=E_{\beta}$. The first term in Eq. (2.1a) and the second term in

Eq. (5.1) have the same form and describe the coupling with non-diagonal matrix elements generated by one-electron hopping between isolated states. The remaining terms in both equations look differently. However, one can easily realize that in the case of coupled dots the only possible transitions for diagonal matrix elements are those corresponding to $\gamma=\alpha$. Then the third term of Eq. (5.1) becomes

$$
\sum_{\delta} 2 \pi \rho \Omega_{\alpha \rightarrow \delta} \Omega_{\delta \rightarrow \alpha} \sigma_{\alpha \alpha}=\sigma_{\alpha \alpha} \sum_{\delta} \Gamma_{\alpha \rightarrow \delta}
$$

It coincides with the second ("dissipative") term of the Bloch rate equation (2.1a). The last term in Eq. (5.1) describes the reverse process, i.e. all possible conversions of the states $\gamma$, $\delta$ into the state $\alpha$. Again, this term turns into the third term of Eq. (2.1a) for $\gamma=\delta$.

The same relation can be traced for the non-diagonal density-matrix elements. We find that the third term of Eq. (5.1), which describe all possible decays of the states $\alpha$ and $\beta$, reproduce the third term in Eq. 2.1b) for $\gamma=\beta$ and $\delta=\alpha$, respectively. The fourth term in Eq. (5.1) describes the reverse process, i.e. the conversion of $\sigma_{\gamma \delta} \rightarrow \sigma_{\alpha \beta}$ produced by two consecutive hoppings through continuum state media. In particular, if $\Omega_{\gamma \rightarrow \alpha}=\Omega_{\delta \rightarrow \beta}$, these terms reproduce the last term of Eq. (2.1b), where $\Gamma_{\gamma \delta \rightarrow \alpha \beta}=2 \pi \rho \Omega_{\gamma \rightarrow \alpha} \Omega_{\delta \rightarrow \beta}$.

\section{SUMMARY}

In this paper we derived quantum rate equations, which provide the most simple and transparent way for a description of the both coherent and incoherent electron transport in quantum dot systems. In the beginning we considered a system of two quantum dots linked by a ballistic channel and connected with the emitter and collector reservoirs. Starting with the many-particle wave function and integrating out the continuum states, we have obtained the equations of motion for the density submatrix of the two-dot system in the occupation number representation. In spite of the quantum dots might be away one from another, we found that the diagonal density-matrix elements are still coupled with the nondiagonal density-matrix elements, similar to Bloch equations for double-well systems. The 
essential difference, however, is that the diagonal matrix elements in the Bloch equations are coupled with the imaginary part of the non-diagonal density-matrix elements, while for the separated wells the corresponding terms are coupled with the real part of the non-diagonal density-matrix elements.

As expected, the coupling between diagonal and non-diagonal matrix elements generated quantum coherent effects in electron transport. For instance, we found that due to destructive quantum interference the dc current in separated dots is reduced when the barrier penetrability increases. This effect would survive even if the dots are largely separated, providing that they are linked by ideal one-dimensional ballistic channels.

In spite of the quantum coherence effects found in dc current, the same system of separated dot, detached from the emitter and collector reservoirs does not show any quantum oscillations. It is quite different from a coupled-dot system with aligned levels, in which an electron oscillates between the dots.

The rate equations for two separated quantum dots are extended to a general case of multi-dot system, in which the dots are either directly coupled, or interconnected via ballistic channels. These new rate equations generalize the well-known Bloch equations, describing time-evolution of the density-matrix of coupled multi-well systems in the presence of a dissipative media. We thus expect that the applicability of our generalized rate equations is not restricted by quantum transport in multi-dot system only, but these equations would be very useful for various physical problems where quantum coherence and classical dissipation effects do interplay.

\section{ACKNOWLEDGMENTS}

I thank Yu. Nazarov for valuable discussions. 


\section{APPENDIX A: DERIVATION OF RATE-EQUATIONS FOR SEPARATED DOTS}

We demonstrate of how the sum over continuum states in the density matrix, Eqs. (3.3). can be performed analytically leading to the rate equations for two-dot systems. In order to simplify the algebraic transformations we use the Laplace transform for the Shrödinger equation $i|\dot{\Psi}(t)\rangle=\mathcal{H}|\Psi(t)\rangle$. Then the amplitudes $b(t)$ in the wave function (3.2) are replaced by their Laplace transform

$$
\tilde{b}(E)=\int_{0}^{\infty} e^{i E t} b(t) d t
$$

Substituting Eq. (3.2) into the Shrödinger equation we obtain an infinite set of coupled equations for the amplitudes $\tilde{b}(E)$ :

$$
\begin{aligned}
& E \tilde{b}_{0}(E)-\sum_{l} \Omega_{l} \tilde{b}_{1 l}(E)=i \\
& \left(E+E_{l}-E_{1}\right) \tilde{b}_{1 l}(E)-\Omega_{l} \tilde{b}_{0}(E)-\sum_{m} \Omega_{m} \tilde{b}_{l m}(E)=0 \\
& \left(E+E_{l}-E_{m}\right) \tilde{b}_{l m}(E)-\Omega_{m} \tilde{b}_{1 l}(E)-\bar{\Omega}_{m} \tilde{b}_{2 l}(E)-\sum_{l^{\prime}} \Omega_{l^{\prime}} \tilde{b}_{12 l l^{\prime}}(E)=0 \\
& \left(E+E_{l}-E_{2}\right) \tilde{b}_{2 l}(E)-\sum_{m} \bar{\Omega}_{m} \tilde{b}_{l m}(E)-\sum_{r} \Omega_{r} \tilde{b}_{l r}(E)-\sum_{l^{\prime}} \Omega_{l^{\prime}} \tilde{b}_{12 l l^{\prime}}(E)=0 \\
& \left(E+E_{l}+E_{l^{\prime}}-E_{1}-E_{2}-U_{12}\right) \tilde{b}_{12 l l^{\prime}}(E)-\Omega_{l^{\prime}} \tilde{b}_{2 l}(E)+\Omega_{l} \tilde{b}_{2 l^{\prime}}(E)- \\
& \quad-\sum_{m} \bar{\Omega}_{m} \tilde{b}_{1 l l^{\prime} m}(E)-\sum_{m} \Omega_{m} \tilde{b}_{2 l l^{\prime} m}(E)-\sum_{r} \Omega_{r} \tilde{b}_{1 l l^{\prime} r}(E)=0
\end{aligned}
$$

Notice that due to the Pauli principle an electron can return back only into unoccupied states of the emitter. As a result, the summation over the emitter states does not appear in the corresponding terms of Eqs. (A2) (in the second term of Eq. (A2b), in the second and the third terms of Eq. (A20), and so on).

Eqs. (A2) can be substantially simplified. Let us replace the amplitude $\tilde{b}$ in the term $\sum \Omega \tilde{b}$ of each of the equations by its expression obtained from the subsequent equation. For example, substitute $\tilde{b}_{1 l}(E)$ from Eq. (A2b) into Eq. (A2a). We obtain

$$
\left[E-\sum_{l} \frac{\Omega_{L}^{2}\left(E_{l}\right)}{E+E_{l}-E_{1}}\right] \tilde{b}_{0}(E)-\sum_{l, m} \frac{\Omega_{L}\left(E_{l}\right) \Omega_{M}\left(E_{m}\right)}{E+E_{l}-E_{1}} \tilde{b}_{l m}(E)=i
$$


where $\Omega_{l} \equiv \Omega_{L}\left(E_{l}\right)$ and $\Omega_{m} \equiv \Omega_{M}\left(E_{m}\right)$. Since the states in the reservoirs are very dense (continuum), one can replace the sums over $l$ and $m$ by integrals, for instance $\sum_{l} \rightarrow$ $\int \rho_{L}\left(E_{l}\right) d E_{l}$, where $\rho_{L}\left(E_{l}\right)$ is the density of states in the emitter. Then the first sum in Eq. (A3) becomes an integral which can be split into a sum of the singular and principal value parts. The singular part yields $-i \Theta\left(E_{F}^{L}+E-E_{1}\right) \Gamma_{L} / 2$, where $\Gamma_{L}=2 \pi \rho_{L}\left(E_{1}\right)\left|\Omega_{L}\left(E_{1}\right)\right|^{2}$ is the level $E_{1}$ partial width due to coupling to the emitter. Let us assume that $E_{F}^{L} \gg E_{1} \gg$ $E_{F}^{M}$, i.e. the energy level is deeply inside the band. In this case the integration over $E_{l(m)^{-}}$ variables can be extended to $\pm \infty$. As a result, the theta-function can be replaced by one, and the principal part is merely included into redefinition of the energy $E_{1}$. The second sum (integral) in Eq. (A3) proves to be negligible small. Indeed, let us replace $\tilde{b}_{l m} \rightarrow \tilde{b}\left(E_{l}, E_{m}, E\right)$, and assume weak energy dependence of $\Omega$ on $E_{l}$. Then one finds from Eqs. (A2) that the poles of the integrand in the $E_{l}$-variable are on one side of the integration contour, and therefore this term vanishes. In general, any terms of the type $\int \cdots d E_{s} \cdots \tilde{b}\left(\cdots, E_{s}, \cdots\right)(E+$ $\left.\cdots \pm E_{s}\right)^{-1} \rightarrow 0$, whenever the integration over the $E_{s}$-variable can be extended to $\pm \infty$. We shall imply this property in all subsequent derivations. Notice that these results are valid also for non-zero temperature, providing that $T \ll E_{F}^{L}-E_{1,2}, E_{1,2}-E_{F}^{M}$.

Now we apply analogous considerations to the other equations of the system (A2). It follows from Eqs. (A2) that the Coulomb repulsion just shifts the energy levels in Eq. (A3), $E_{1,2} \rightarrow E_{1,2}+U_{i j}$. If the shifted level is outside the band, the singular part of the integral vanishes and therefore the corresponding width $\Gamma$ is replaced by zero. If, however, the shifted energy stands deeply inside the band, all the previous treatment remains the same. A problem appears only if the shifted energy in near the Fermi level. This case is not considered here. Finally we arrive at the following set of equations:

$$
\begin{aligned}
& \left(E+i \Gamma_{L} / 2\right) \tilde{b}_{0}=i \\
& \left(E+E_{l}-E_{1}+i \Gamma_{M} / 2\right) \tilde{b}_{1 l}-\Omega_{l} \tilde{b}_{0}+i \pi \rho_{M} \Omega_{M} \bar{\Omega}_{M} \tilde{b}_{2 l}=0 \\
& \left(E+E_{l}-E_{m}+i \Gamma_{L} / 2\right) \tilde{b}_{l m}-\Omega_{m} \tilde{b}_{1 l}-\bar{\Omega}_{m} \tilde{b}_{2 l}=0 \\
& \left(E+E_{l}-E_{2}+i \Gamma_{L}^{\prime} / 2+i \bar{\Gamma}_{M} / 2+i \Gamma_{R} / 2\right) \tilde{b}_{2 l}+i \pi \rho_{M} \Omega_{M} \bar{\Omega}_{M} \tilde{b}_{1 l}=0
\end{aligned}
$$


$\left(E+E_{l}+E_{l^{\prime}}-E_{1}-E_{2}-U_{12}+i \Gamma_{M}^{\prime} / 2+i \bar{\Gamma}_{M}^{\prime} / 2+i \Gamma_{R}^{\prime} / 2\right) \tilde{b}_{12 l l^{\prime}}-\Omega_{l^{\prime}} \tilde{b}_{2 l}+\Omega_{l} \tilde{b}_{2 l^{\prime}}=0$,

where $\Gamma_{M}=2 \pi \rho_{M}\left(E_{1}\right)\left|\Omega_{M}\left(E_{1}\right)\right|^{2}$ and $\bar{\Gamma}_{M}=2 \pi \rho_{M}\left(E_{2}\right)\left|\bar{\Omega}_{M}\left(E_{2}\right)\right|^{2}$ are the partial widths of the levels $E_{1}$ and $E_{2}$ respectively due to coupling to the ballistic channel and $\rho_{M}$ is the density of states in the ballistic channel. $\Gamma_{R}=2 \pi \rho_{R}\left(E_{2}\right)\left|\Omega_{R}\left(E_{2}\right)\right|^{2}$ is the level $E_{2}$ partial width due to coupling to the collector. The Coulomb interdot repulsion $U_{12}$ just modifies the corresponding width ( $\Gamma$ is replaced by $\Gamma^{\prime}$, evaluated at the energy $\left.E_{1,2}+U_{12}\right)$ whenever both dots are occupied [17]. A similar modification would take place if we include the Coulomb repulsion between electrons inside the dots and the ballistic channel. In the following we assume that widths are weakly dependent on the energy, so $\Gamma^{\prime}=\Gamma$ everywhere.

The density matrix elements, Eqs. (3.3), are directly related to the amplitudes $\tilde{b}(E)$ through the inverse Laplace transform

$$
\sigma^{(k, n)}(t)=\sum_{l \ldots, m, \ldots, r \ldots} \int \frac{d E d E^{\prime}}{4 \pi^{2}} \tilde{b}_{l \cdots m \cdots r \ldots}(E) \tilde{b}_{l \cdots m \ldots r \ldots}^{*}\left(E^{\prime}\right) e^{i\left(E^{\prime}-E\right) t}
$$

Using this equation one can transform Eqs. (A4) for the amplitudes $\tilde{b}(E)$ into differential equations for the probabilities $\sigma^{(k, n)}(t)$. Consider, for instance, the term $\sigma_{b b}^{(0,0)}(t)=$ $\sum_{l}\left|b_{1 l}(t)\right|^{2}$, Eq. (3.3b). Multiplying Eq. ( conjugated equation with the interchange $E \leftrightarrow E^{\prime}$ we obtain

$$
\begin{aligned}
& \int \frac{d E d E^{\prime}}{4 \pi^{2}} \sum_{l}\left\{\left(E^{\prime}-E-i \Gamma_{M}\right) \tilde{b}_{1 l}(E) \tilde{b}_{1 l}^{*}\left(E^{\prime}\right)-\Omega_{l}\left[\tilde{b}_{1 l}(E) \tilde{b}_{0}^{*}\left(E^{\prime}\right)-\tilde{b}_{1 l}^{*}\left(E^{\prime}\right) \tilde{b}_{0}(E)\right]\right. \\
&\left.-i \pi \rho_{M} \Omega_{M} \bar{\Omega}_{M}\left[\tilde{b}_{1 l}(E) \tilde{b}_{2 l}^{*}\left(E^{\prime}\right)+\tilde{b}_{1 l}^{*}\left(E^{\prime}\right) \tilde{b}_{2 l}(E)\right]\right\} e^{i\left(E^{\prime}-E\right) t}=0 .
\end{aligned}
$$

Substituting

$$
\tilde{b}_{1 l}(E)=\frac{\Omega_{l} \tilde{b}_{0}(E)-i \pi \rho_{M} \Omega_{M} \bar{\Omega}_{M} \tilde{b}_{2 l}(E)}{E+E_{l}-E_{1}+i \Gamma_{M} / 2}
$$

into Eq. (A6) we can carry out the $E, E^{\prime}$-integrations thus obtaining

$$
\dot{\sigma}_{b b}^{(0,0)}=-\Gamma_{M} \sigma_{b b}^{(0,0)}+\Gamma_{L} \sigma_{a a}^{(0,0)}-\pi \rho_{M} \Omega_{M} \bar{\Omega}_{M}\left(\sigma_{b c}^{(0,0)}+\sigma_{c b}^{(0,0)}\right),
$$

It corresponds to Eq. (3.5a) for $k=n=0$. Applying the same procedure to each of equations (A4d) we obtain the Bloch-type equations, (3.5) for the density matrix element $\sigma^{(k, n)}(t)$. 


\section{REFERENCES}

[1] D.V. Averin and K.K. Likharev, in Mesoscopic Phenomena in Solids, edited by B. Altshuler, P.A. Lee, and R.A. Webb (Elsevier, Amsterdam, 1991).

[2] R.J. Haug, J.M. Hong, and K.Y. Lee, Sirf. Sci. 263, 415 (1992).

[3] N.C. van der Vaart, S.F. Godijn, Y.V. Nazarov, C.J.P.M. Hartmans, J.E. Mooij, L.W. Molenkamp, and C.T. Foxon, Phys. Rev. Lett. 74, 4702 (1995).

[4] F.R. Waugh, F.R. Waugh, M.J. Berry, D.J. Mar, R.M. Westervelt, K.L. Campman and A.C. Gossard, Phys. Rev. Lett. 75, 4702 (1995).

[5] Bryant, Phys. Rev. B 44, 3064 (1991).

[6] S.A. Gurvitz, Phys. Rev. B 44, 11924 (1991).

[7] A.N. Korotkov, D.V. Averin, and K.K. Likharev, Phys. Rev. B 49, 7548 (1994).

[8] C.A. Stafford and S. Das Sarma, Phys. Rev. Lett. 72, 3590 (1994).

[9] L.I. Glazman and K.A. Matveev, Pis'ma Zh. Eksp. Theor. Fiz. 48, 403 (1988) [JETP Lett. 48, 445 (1988)].

[10] D.V. Averin and A.N. Korotkov, Zh. Eksp. Teor. Fiz. 97, 1661 (1990) [Sov. Phys.-JETP 70, $937(1990)$.

[11] C.W.J. Beenakker, Phys. Rev. B 44,1646 (1991).

[12] J.H. Davies, S. Hershfield, P. Hyldgaard, and J.W. Wilkins, Phys. Rev. B 47, 4603 (1993).

[13] G. Klimeck, G. Chen, and S. Datta, Phys. Rev. B 50, 2316 (1994)

[14] G. Chen, G. Klimeck, S. Datta, G. Chen, and W.A. Goddard, Phys. Rev. B 50, 8035 (1994).

[15] Yu.V. Nazarov, Physica B 189, 57 (1993). 
[16] S.A. Gurvitz, H.J. Lipkin, and Ya.S. Prager, Phys. Lett. A 212, 91 (1996).

[17] S.A. Gurvitz and Ya.S. Prager, Phys. Rev. B53, 15932 (1996).

[18] S.A. Gurvitz, Phys. Rev. B, 15 Dec. (1997).

[19] C. Cohen-Tannoudji, J. Dupont-Roc, and G. Grynberg, Atom-Photon Interactions: Basic Processes and Applications (Wiley, New York, 1992).

[20] J. Rammer and H. Smith, Rev. Mod. Phys. 58, 323 (1986).

[21] The results are valid also for non-zero temperature providing that $T \ll E_{F}^{L}-E_{1,2}, E_{1,2}-$ $E_{F}^{R}$. Otherwise, the average over the reservoir states would enter explicitly in the equations.

[22] S. Bjornholm and J.E. Lynn, Rev. Mod. Phys. 52, 725 (1980).

[23] S.A. Gurvitz, and M.S. Marinov, Phys. Rev. A40, 2166 (1989).

[24] S.A. Gurvitz, I. Bar-Joseph and B. Deveaud, Phys. Rev. B 43, 14 703, (1991).

[25] G. Cohen, S.A. Gurvitz, I. Bar-Joseph, B. Deveaud, P. Bergman, and A. Regreny, Phys. Rev. B 47, 16 012, (1993).

[26] A. Beige and G.C. Hegerfeldt, Phys. Rev. A 53, 53 (1996), and references therein.

[27] A.N. Korotkov, unpublished. 